\title{
Eficacia del Articulador Semiajustable en el Diagnóstico de Maloclusión de Pacientes del Centro Ortodóncico de Tacna, 2010.
}

\author{
Efficacy of Semi-adjustable Articulator using in the Diagnosis of \\ Malocclusion of Patients in the Ortodoncic Center of Tacna, \\ 2010 .
}

${ }^{1}$ Luis Alberto Alarico Cohaila

\begin{abstract}
RESUMEN:
El objetivo del estudio, se centró en determinar la eficacia del articulador semiajustable frente a un método convencional para el diagnóstico ortodóncico, en el Centro Ortodóncico de la ciudad de Tacna, en el año 2010. Se aplicó el arliculador semiajustable para el diagnóstico de maloclusiones sagitales, verticales y transversales a 32 pacientes de ambos sexos. El grupo de estudio también fue el grupo de comparación (control). Entre los principales resultados, se tiene que el articulador presenta una eficacia total promedio para el diagnóstico ortodóncico de 32 y una ineficacia promedio de 0; mientras que el método convencional presenta una eficacia promedio 29.66 para el diagnóstico de las maloclusiones transversales: 22.5 para las maloclusiones verticales y de 21.33 para las sagitales, y una ineficacia total promedio de 10.66 para el diagnóstico de las malochusiones sagitales. Se concluye, que la aplicación del articulador semiajustable en el grupo de estudio, cleterminó una eficacia én el diagnóstico del $100 \%$.
\end{abstract}

Palabras clave: articulador: diagnóstico, maloclusiones.

\section{ABSTRACT:}

The objective of study was focused on determining the efficacy of semi-adjustable articulator in contrast to a conventional method, in reference to orthodontic treatment, in the Orthodontic Center of Tacna City, during the year 2010. The semi adjustable articulator was administered to diagnostic sagital, vertical, and transversal malocclusions in thirty two male and female patients. The study group was also the comparison group (control). The main results were: the articulator presents an efficacy average for the orthodontic diagnosis of thirty two and a zero average efficac $y$, whereas the conventional method presents a twenty nine, sixty six average efficacy for the diagnosis of transversal malocclusions; twenty two, five related to vertical malocclusions and twenty one, thirty three for sagital malocclusions and an average efficacy of ten, sixty six in reference to sagital malocclusions diagnosis. As a conclusion, the application of the semi-adjustable articulator in the study group determine a one hundred percent efficacy in relation to the diagnosis.

Key Words: articulator; diagnosis, malocclusions.

\footnotetext{
' Doctor en Ciencias de la Salud, Maestria en Docencia Universitaria. Especialista en Ortodoncia y Ortopedia Dentomaxilar. Cirujano Dentista. Facultad de Ciencias de la Salud. Universidad Nacional Jorge Basadre Grohmann
} 


\section{INTRODUCCIÓN}

La correcta función del Sistema Estomatognático, sus funciones básicas como fonación, deglución y masticación, es un área de interés para todas las especialidades odontológicas. En este campo, actualmente se sigue polemizando en vista de que no hay consenso entre los profesionales sobre la estrategia óptima para rehabilitar a nuestros pacientes en relación céntrica (RC)(Fig. 1).

Durante nuestros estudios en la especialidad de
Ortodoncia, tanto en la Universidad de Chile como en el Curso Continuado con el Dr. Ronald Roth, así como en la práctica privada, se han presentado problemas en el tratamiento ortodóncico de algunos pacientes en los que la discrepancia entre OC y RC era significativa. (Fig. 2). De allí nuestra inquietud por incluir, en la etapa diagnóstica, el análisis de la oclusión del paciente, y esto sólo se puede hacer de manera correcta con el uso de articuladores semiajustables que nos permitan determinar la verdadera relación céntrica del paciente y su coincidencia o no con la oclusión céntrica.(Fig.3 y Fig.4)
Método Convencional

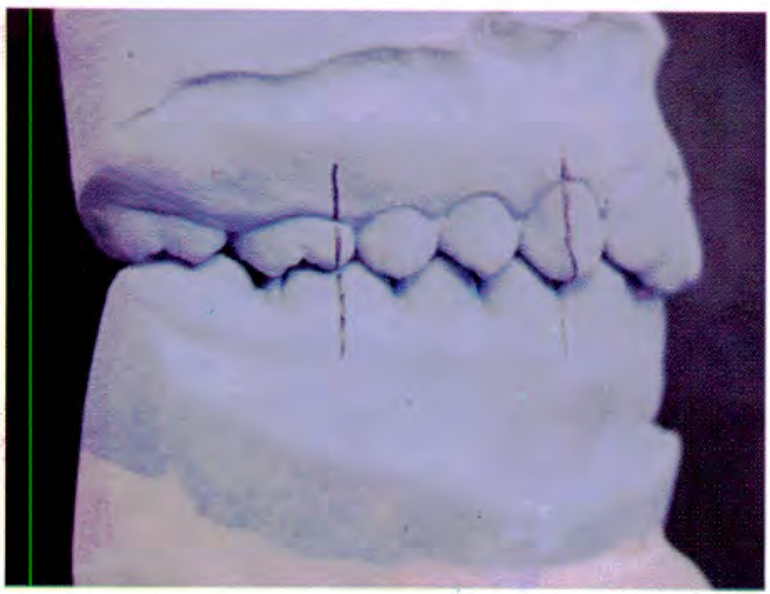

Fig. 1 Modelos del paciente en oclusión habitual $(\mathrm{OH})$ en una vista lateral derecha en donde se aprecia la relación molar, relación canina y el over jet.

$\mathrm{Al}$ respecto, Martino $\mathrm{M}$, refiere que el montar o no los casos de ortodoncia en articuladores semiajustables, ha sido fuente de debate por décadas en la especialidad. Sostiene, que en una encuesta realizada a sus suscriptores por el Journal Of Clinical Orthodontics en el 2001, indicó que mientras el $21 \%$ montaba sus modelos de manera rutinaria, el $44 \%$ lo hacía
Con Articulador Semiajustable

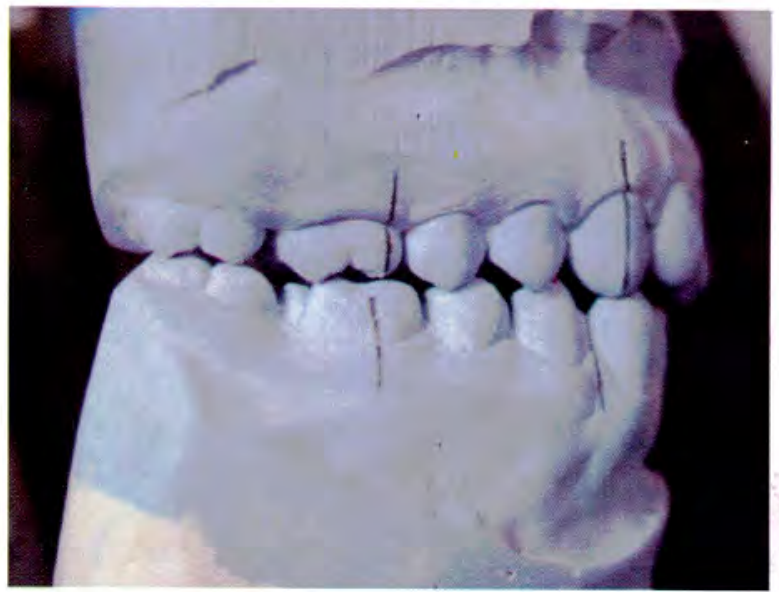

Fig. 2 Modelos del paciente en relación céntrica $(R C)$ en una vista lateral derecha en donde se aprecia el cambio en la relación molar, canina y over jet.

las consideraciones gnatológicas y la prevención de desórdenes témporomandibulares (DTM) las razones principales para hacerlo. No hay duda de la importancia del uso del articulador en otras áreas de odontología, como la prótesis y la cirugía ortognática, pero su utilidad en ortodoncia es controversial. (34)

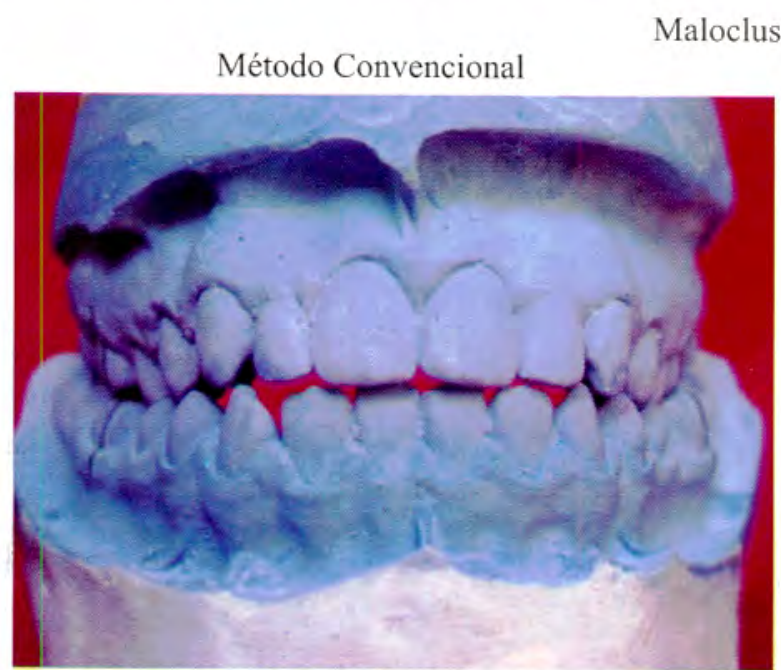

Fig. 3 Modelos del paciente en oclusión habitual $(\mathrm{OH})$ en una vista frontal en donde se aprecia el tipo de mordida en sentido vertical (over bite)

\section{Con Articulador Semiajustable}

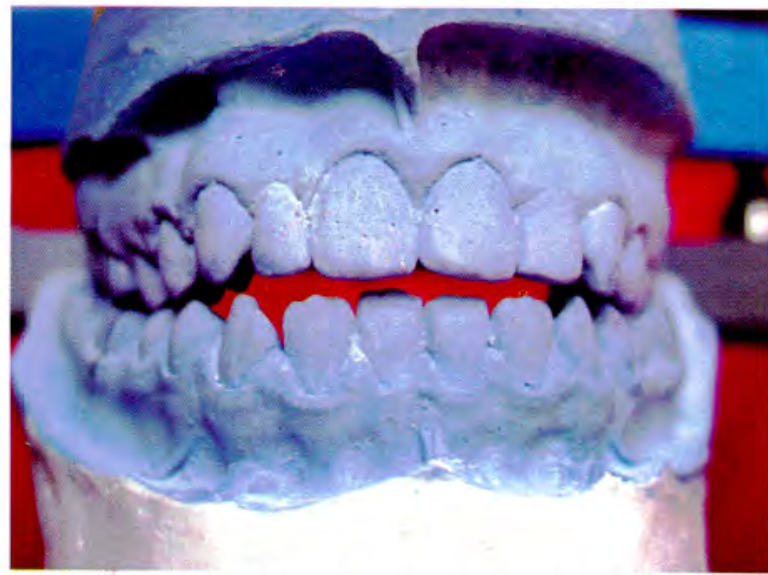

Fig. 4 Modelos del paciente en relación céntrica (RC) en una vista frontal en donde se aprecia el cambio en la mordida, en este caso se acentúa la mordida abierta y por consiguiente el over bite disminuye 
Método Convencional

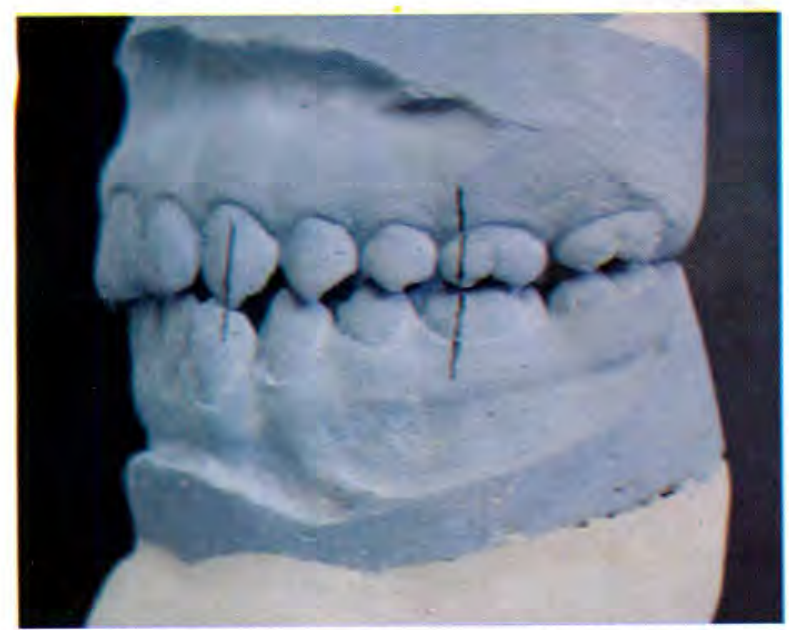

Fig. 5 Modelos del paciente en oclusión habitual $(\mathrm{OH})$ en una vista lateral izquierda para apreciar el grado de mordida en sentido transversal.

En la odontología, como en todas las ciencias de la salud, las decisiones deben realizarse utilizando juiciosamente la mejor evidencia actual disponible. Es un hecho que la mejor evidencia es la de tipo científico, pero en ciertos aspectos de nuestra profesión ésta es escasa, ya sea por una limitada investigación o por dificultades técnicas en la experimentación, lo que muchas veces determina que las decisiones se basen en la experiencia o la casuística y no en la evidencia científica.

En tal sentido, la línea de investigación está centrada en el uso del articulador, como un elemento que favorece un diagnóstico ortodóncico no sólo oportuno, sino correcto, frente a un método convencional.

\section{Objetivo}

Determinar la eficacia del articulador semiajustable para el diagnóstico de maloclusión respecto al método convencional en los pacientes del Centro Ortodóncico de Tacna, en el año 2010.

\section{MATERIALY MÉTODOS}

El presente estudio corresponde a un diseño experimental, ya que se trata de "una indagación empírica y sistemática, en la cual el científico tiene el control directo sobre la variable independiente"

Según el corte temporal, es un diseño longitudinal ya que implica la recolección de los datos después de la manipulación del estímulo en dos oportunidades; y según la visión temporal es un estudio prospectivo, ya que se inician con la observación de ciertas causas presumibles y avanzan longitudinalmente en el tiempo a fin de observar sus consecuencias, en el caso particular, el estudio se desarrolló desde el mes de julio a diciembre del año 2010.

Participaron 32 modelos de estudio que fueron
Con Articulador Semiajustable

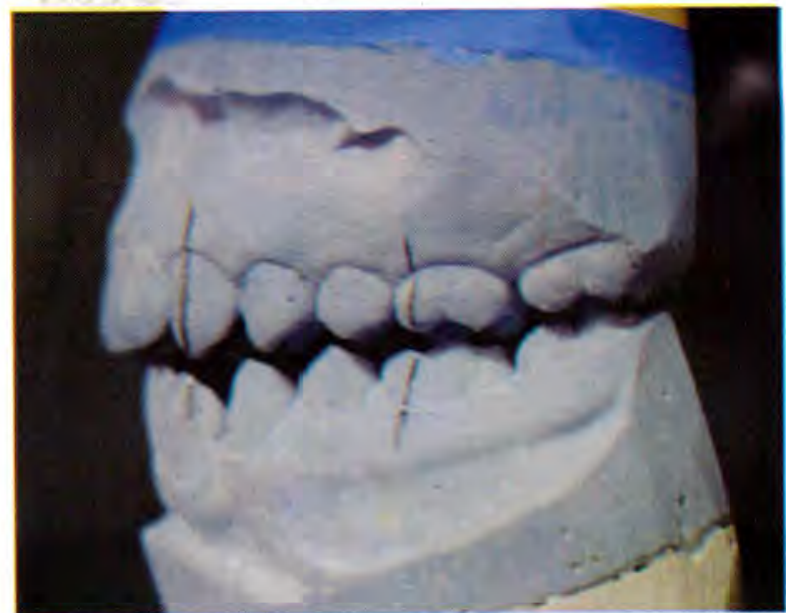

Fig. 6 Modelos del paciente en relación céntrica (RC) en una vista lateral izquierda para apreciar el cambio en la mordida en sentido transversal

analizados con el articulador semiajustable y con el método convencional. (Fig.5 y Fig. 6). Esto es, se utilizó un grupo observado en dos tiempos. En el primer tiempo será experimental y en un segundo momento será el grupo control. Para vaciar la información obtenida mediante el examen clínico y establecido el diagnóstico, se utilizó una ficha de recolección de datrs cuyo contenido fue validado por expertos. Se utilizaron los siguientes materiales y equipos: a) Modelos articulados y no articulados de los pacientes integrantes de la muestra, b) instrumental médico: articuladores, espejo, pinza, explorador, c) Yeso París, cera Delar azul, cera rosada, godiva.

\section{RESULTADOS}

En cuanto a la valoración de la eficacia del articulador versus método convencional en el diagnóstico de maloclusiones sagitales, verticales y transversales, se debe resaltar que para el primero la eficacia promedio es de 32; mientras que para el segundo, la eficacia promedio para las maloclusiones sagitales es del 21.33; para las maloclusiones verticales es del 22.5 y para las transversales es de 29.66; resultados que indican una eficacia diferente, con evidencia en cuanto a una mayor sensibilidad de clasificar a un paciente como portador de una maloclusión, y que la prueba diagnóstica sea positiva cuando realmente el paciente presenta la patología.(Fig. 7)

Respecto a la discrepancia entre oclusión habitual y relación céntrica ( $\mathrm{OH}$ y $\mathrm{RC})$, los resultados evidencian una discrepancia en 28 casos, lo que representa el $87.50 \%$ cuando se usa articulador, lo que significa que existe un número mínimo de casos en los cuales existe una coincidencia entre $\mathrm{OH}$ y RC. Sin embargo, se subraya, que en muchos casos, la discrepancia se ve favorecida por interferencias dentarias, las que atendiendo a su magnitud se puede traducir en un trauma oclusal o deslizamientos mandibulares. 
En nuestro estudio se encontraron discrepancias entre oclusión habitual y relación céntrica que deberian tenerse en cuenta al momento del diagnóstico para no errar en el tratamiento y poder obtener resultados exitosos.

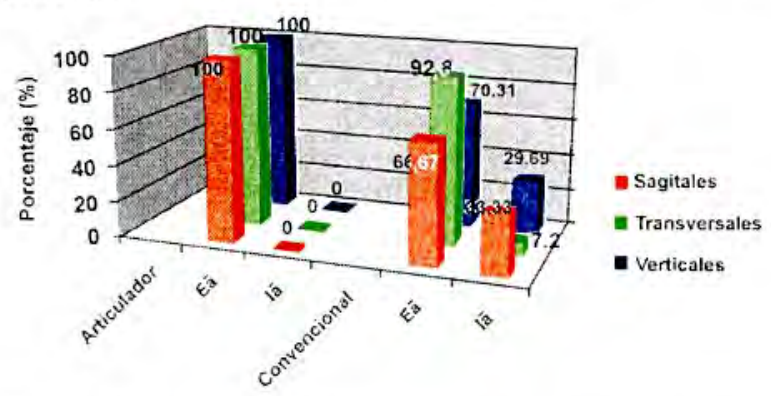

Fig. 7 Eficacia Total del Articulador y del método convencional en el diagnostico de maloclusiones

\section{DISCUSIÓN}

La presente investigación, tuvo como propósito central verificar la eficacia del articulador semiajustable frente al uso del método convencional en el diagnóstico de maloclusiones dentarias, ya que la importancia del diagnóstico en ortodoncia es fundamental. En la práctica ortodóncica, el diagnóstico representa el $75 \%$ del tratamiento, mientras que el plan de tratamiento supone el $20 \%$ y el $5 \%$ restante descansa en la biomecánica.

Al realizar el análisis descriptivo de las caracteristicas demográficas, edad y sexo de los pacientes que participaron en el estudio, encontramos que el sexo que predomina es el femenino, ya que representa el $62.50 \%$ de los sujetos de estudio y el grupo etáreo que predomina es el de 10 a 20 años. Esta proporción alta en el grupo de pacientes jóvenes adultos y adolescentes y de sexo femenino, encuentra una explicación en el imperativo que la sociedad contemporánea impone de ofrecer una apariencia deseable en ambos sexos, pero que ineludiblemente pareciera preocupar en mayor cuantia al sexo femenino.

\section{V.CONCLUSIONES}

1. El articulador semiajustable es más eficaz en el diagnóstico ortodóncico de maloclusiones sagitales respecto a la relación molar que el procedimiento convencional $(\mathrm{P}=0.001)$.

2. El articulador semiajustable es más eficaz en el diagnóstico ortodóncico de maloclusiones sagitales respecto a la relación canina que el procediniento convencional $(\mathrm{P}=0.001)$.

3. El articulador semiajustable es más eficaz en el diagnóstico ortodóncico de maloclusiones sagitales respecto al Over Jet que el procedimiento convencional $(\mathrm{P}=0.001)$.

4. El articulador semiajustable es más eficaz en el diagnóstico ortodóncico de maloclusiones verticales respecto a la Mordida Abierta que el procedimiento convencional $(\mathrm{P}=0.001)$.
5. El articulador semiajustable es más eficaz en el diagnóstico ortodóncico de maloclusiones verticales respecto a la Sobremordida que el procedimiento convencional $(\mathrm{P}=0.001)$.

6. El articulador semiajustable es más eficaz en el diagnóstico ortodóncico de maloclusiones verticales respecto al Bis a Bis que el procedimiento convencional $(\mathrm{P}=0.001)$.

7. El articulador semiajustable es más eficaz en el diagnóstico ortodóncico de maloclusiones verticales respecto al Over Bite que el procedimiento convencional $(\mathrm{P}=0.001)$.

8. El articulador semiajustable es más eficaz en el diagnóstico ortodóncico de maloclusiones transversales respecto a la Mordida Cruzada que el procedimiento convencional $(\mathrm{P}=0.001)$.

9. El articulador semiajustable es más efícaz en el diagnóstico ortodóncico de maloclusiones transversales respecto a la Mordida en Vestibulo Oclusión que el procedimiento convencional $(\mathrm{P}=0.001)$.

10. El articulador semiajustable es más eficaz en el diagnóstico ortodóncico de maloclusiones transversales respecto a la Desviación de la Línea Media que el procedimiento convencional $(\mathrm{P}=0.001)$.

11. El articulador presenta una eficacia total promedio de 32 y una ineficacia total promedio de 0 para el diagnóstico de maloclusiones sagital, transversal y verticales; y el método convencional presenta una eficacia total promedio de 29.66 para el diagnóstico de las maloclusiones transversales, la que es alta respecto a las demás y la ineficacia total promedio más alta fue de 10.66 para el diagnóstico de las maloclusiones sagitales.

\section{REFERENCIAS BIBLIOGRÁFICAS}

1. Academy of Denture Prosthetics, citado por Serrano Belmonte I, Forcén Báez A. Ruiz Navas MT. RoyoVillanova Pérez ML. Estudio del triángulo de Bonwill y su aplicación a los articuladores semiajustables (1). Rev EurOdontoestomatol 2001: 13 (3): 123-30(a), 1977

2. Alarico Cohaila, Luis A. Diagnóstico Ortodóncico. Pág. 75. Editorial Graficom. Universidad Católica Santa Maria. Arequipa-Perú. 2007

3. Alarico Cohaila, Luis A. Ventajas del uso del articulador semiajustable en el diagnóstico ortodóncico de pacientes del Centro Ortodóncico de Tacna-2007. Tesis para optar el grado de Doctor en Ciencias de la Salud, Arequipa.

4. Alonso, A. A. Oclusión y Diagnóstico en rehabilitación Oral. Pág. 498. Editorial Médica Panamericana. 2000.

5. ALVES, R.J. Actualización en Ortodoncia y Ortopedia Funcional de los Maxilares. Pág. 133. Editorial Artes Médicas Latinoamérica. Sao PauloBrasil. 2002 
6. Ayala J. Rol de los articuladores en Ortodoncia y Odontologia. Rev.Chilena de Ortodoncia. 14: $4-21$, 1997.

7. Barbosa, J. A. Diagnóstico Ortodóncico en relación Céntrica. En INTERLANDI, S. Ortodoncia: Bases para la iniciación. Cap. 16. pág. 265-283.2006

8. Del Río Highsmith J. Guías clinicas de las terapéuticas en Odontología Integrada para adultos.Edit. Lerko Print. Madrid, 1998.

9. Daniel, W. Estadistica con aplicaciones a las Ciencias Sociales y a la Educación, Edit. Mc Graw Hill, Bogotá. 1995.

10. Dos Santos, José. Gnatología, Principios y Conceptos. Ed. Actualidades Medico- Odontológicas, Latinoamérica, 1992

11. Echeverri, Enrique . SENCHERMAN K. Neurofisiología de la Oclusión. Editorial Monserrate. Bogotá-Colombia. 1984.

12. Espinosa de la Sierra, Raúl. Diagnóstico Práctico de Oclusión. Pág. 83. Editorial Médica Panamericana. México, D.F. 1995.

13. Howat, Alison. Capp, Nicholas y Barrett, Vincent. Oclusión y Maloclusión. Editorial Mosby Year Book. Pág. 28. Gran Bretaña-1997.

14. Kerlinger $F$ (1988) Investigación del comportaniento, Edit. McGrawn Hill., México D.F.

15. Fernandez S. Jesús. Manual de Prácticas de Odontopediatría, Ortodoncia y Odontología Preventiva. Editorial Médica Ripano. 2006.

16. Fonseca Balcázar, Franco. La importancia del Diagnóstico en relación céntrica en Ortodoncia. Revista Medicina oral. 6(2): 54-57.2004.

17. Interlandi, Sebastiao. ORTODONCIA: Bases para la iniciación. Pag. 29-34. Editorial Artes Médicas. Sao Paulo-Brasil. 2002

18. Lee R. Estética y su Relación con la Función. En Rufenacht. Claude, Fundamentos de Estética. Cap. 5. P. 2.3 137-210. Publicaciones Quintaessence. 1992.

19. Manns, Arturo E. Manual Práctico de Oclusión dentaria. Pag. 63. Editorial Amolca. Venezuela. 2006.

20. Okeson, J. P. Tratamiento de Oclusión y Afecciones Témporomandibulares. Pág. 124. Editorial Elsevier España. 2003

21. Pabón, Ambrosio, Procedimiento Clínico y de Laboratorio para el montaje de los modelos de estudio en el Articulador Semiajustable Whip Mix 8500 en Prótesis parciales fijas y Removibles. Pág. 3. Universidad de los Andes. Facultad de Odontología. Departamento de Odontología Restauradora. MéridaVenezuela. 2002.
22. Rosado Linares, Larry. Guía Metodológica. Determinación del tamaño de la muestra para la Investigación Cientifica en Salud. Pag. 21-23. Universidad Católica Santa María Facultad de Odontología. Arequipa-Perú. 2005.

23. Roth $R H$. Comunicación personal. Curso Continuado de Ortodoncia, Roth-Williams Center for Functional. Santiago de Chile. 2002-2004.

24. Roth R H. Oclusión Funcional para el Ortodoncista. J CO. 15: 32-5I, 100-23, 174-98,246-65, Ene-Feb-MarAbril. 1981

25. Roth R H. Sistemas de mantenimiento y Dinámica Oclusal. Clinicas Odontológicas de Norteamérica. Ed. Norteamericana. Pág. 761-89.1976.

26. Sapunar, Anka. Diagnóstico y Planificación de Tratamiento ortodóncico basado en los objetivos de la filosofia del Dr. R. Roth. Revista Chilena de Ortodoncia Vol. 19:68-69, 2002.

27. Silva Ruiz, Henry. Utilidad del indicador de posición condilar (IPC) en el diagnóstico y tratamiento de la disfunción de la Articulación Témporomandibular. Pág. 24-25. Universidad Nacional Autónoma de Nicaragua UNAM. MANAGUA. 2004.

28. Tasaky, Prince. Diagnóstico en Ortodoncia: puntos de encuentro entre el respeto del sistema estomatognático con el aspecto facial y la oclusión (Parte IV). Revista Ortodoncia Clínica. 6(4):226-229, 2003.

29. Ustrell i Torrent Josep, Durán Von Arx José. Brau Maire Isabel, Ortodoncia, Edicions Universitan de Barcelona, 2009.

\section{Direcciones Electrónicas}

30. Ferrer, M. Oclusión basada en la evidencia (Partell) No. 153-Nov. 2004-Ciencia.[en línea] Disponible en http://www.gacetadentalcom/articulos.asp? aseccion $=$ ciencia\&avol=2 [ Consulta 21.12.2009].

31. Clark, J.R. Functional Occlusion: II. The Role of Articulators in Orthodontics. [ en línea ] Disponible en ht t p://jorthod. mancy journals.org /cgi/reprint/28/2/173.pdf. [ Consulta 21.12.2009].

32. Clark, J. R.; EVANS, R.D. Functional occlusal relationships in a group of post-orthodontic patients: preliminary findings. [ en línea ] Disponible en http://ejo.oxford journals.org/egi/reprint/20/2/103 [Consulta 1.10.2009].

33. Castillo de Oyagüe R., Del Rio Highsmith J., Sánchez Turrión A., Serrano Madrigal Benjamín, El articulador semiajustable, Madrid [en linea] Disponible en

http://www.salvadorinsignares.com/programaonline/pr ogramarehabilitacion/oclusion/Articuladores.htm [Consulta 11.01.2011]. 
34. Martino Martinez F. Uso de articuladores en ortodoncia: Separando las evidencias cientificas de las observaciones clinicas [en línea] Disponible en

http://webcache.googleusercontent.com/search?hl=es

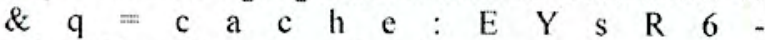
cOv3kJ:http://www.ortodoncia.org.ar/descargas/revist a/143/Ortodoncia $2008 \quad 7$ I 14360 7.pdf +investigaciones+en+ortodonciat++ uso $+\mathrm{de}+$ articuladores\&ct $=\mathrm{clnk} \quad$ [Consulta $01.12 .2011]$.

35. O'malley, A. M.: Milosevic, A. Comparison of three facebow/semi-adjustable articulator systems for planning [ en línea] Disponible en

http:/www.sld.cu/galerias/pdf/sitios/protesis/comparis o n of three face bow - s e m i adjustable_articulator_systems_for_orthognatic [Consulta 3.07.2009].

36. Rinchuse, Donald J. Centric Relation. A historical and contemporary orthodontic perspective[ en linea ] Disponible en http:/jada.ada.org/ [Consulta 13.02.2009].
37. Rinchuse, Donald J. Articulators in Orthodontics (Part 1) [en línea] Disponible en http://www.orthodonticproductsonline.com/issues/arti cles/2007-04_19.asp [Consulta 15.01.2009].

38. Rinchuse, Donald J. Articulators in Orthodontics (Part 2) [ en linea] Disponible en

http://www.orthodonticproductsonline.com/issues/arti cles/2007-06 .08.asp [Consulta 31.12.2008].

\section{Correspondencia:}

Luis Alberto A larico Cohaila

Ciudad Universitaria Fundo "Los Granados"

Calle Miraflores s $/ \mathrm{n}$. Tacna. Perú

luisalarico@gmail.com 